\title{
Relationship between Water Temperature and Bisexual Reproduction Rate in the Rotifer Brachionus plicatilis*1
}

\author{
Akinori Hino*2 and Reijiro HiRANo*2 \\ (Accepted January 20, 1984)
}

\begin{abstract}
Brachiomus plicatilis was cultured individually and successively under four $\left(15,20,25\right.$ and $\left.30^{\circ} \mathrm{C}\right)$ temperature conditions, and the frequency of mictic female production was examined. In order to avoid the density effect derived from accumulation of metabolites, culture medium was renewed at regular intervals proportionally to the metabolic rate which was specific to each temperature.

The hereditary differences in bisexual reproductivity existing among strains could be negligible through experimenting on identical strain. A hundred substrains were made from the same stock, and cultured for 7-8 generations, picking up five offsprings in one generation-one strain. Data from the initial two generations were rejected, because a temporary lowering of mictic female production was observed just after the transference into new environmental condition.

Bisexual reproduction rate increases in lower temperature below $25^{\circ} \mathrm{C}$. There is no significant diferrence between 25 and $30^{\circ} \mathrm{C}$ groups.
\end{abstract}

Rotifers have been very attractive since the last century among biologists because of their worldwide distribution and peculiarity in life cycle. In recent years, they have become indispensable as food organisms in seedling production of aquaculture. Particularly in Japan, several million fish seedlings are annually released in natural waters even by a single prefectural institute. Consequently, many informations have been accumulated particularly on Brachionus plicatilis, supposing a large scale production as in a $100-200 \mathrm{~m}^{3}$ tank. There are also many fundamantal studies on the biology of $B$. plicatilis, such as growth rate in experimental envilonments, filtering rates, morphological variety etc.*3 In addition, the inducing factors of bisexual reproduction have been also studied relating to continuation of parthenogenesis which is prerequisite to massculture. Bisexual reproduction produces male offsprings or only two or three fertilized dormant eggs, which does not contribute to population growth.

On the bisexual reproduction of B. plicatilis, it was reported that high density culture induces relatively high mictic female production..$^{1-8}$ In addition to this external factor, HINo and HIRANO ${ }^{4}$ made clear the preceding action of internal factors that the ability of bisexual reproduction differs among strains and it is acquired as generation passes in a single pure line. In this study the relation between bisexual reproduction rate and temperature was experimented after considering the action of internal factors. Concerning the effect of temperature on the bisexual reproduction of $B$. plicatilis, Imamura et al..$^{5)}$ succeeded in a large scale production of dormant eggs after stimulating through relatively high temperature. The mechanism of dormant egg formation is not, however, completely clear, because many steps must be existing in a population from the beginning of bisexual reproduction till the completion of dormant eggs. This study treats the appearance of a mictic female that is the first step of bisexual reproduction.

\section{Materials and Methods}

B. plicatilis experimented was the same stock as examined in previous studies, ${ }^{3,4)}$ and all individuals belonged to the identical strain which had only one stem mother as the ancestory that had hatched from a fertilized egg. Culture medium was food suspending seawater of which chlorinity was adjusted to $16 \%$. In this study, dry yeast for baking was applied to food without any activation, in order to

* A part of this study was supported by a grant-in-aid for scientific research.

*2 Department of Fisheries, Faculty of Agriculture, The University of Tokyo, Yayoi 1-1, Bunkyo, Tokyo 113, Japan (日野明德・平野礼次郎：東京大学農学部水産学科)。

*3 These are generally reviewed in "Shiomizu tsubo wamushi" (ed. by Japan. Soc. Sci. Fish.), Suisangaku series No. 44, Koseisha Koseikaku, Tokyo, 1983. 


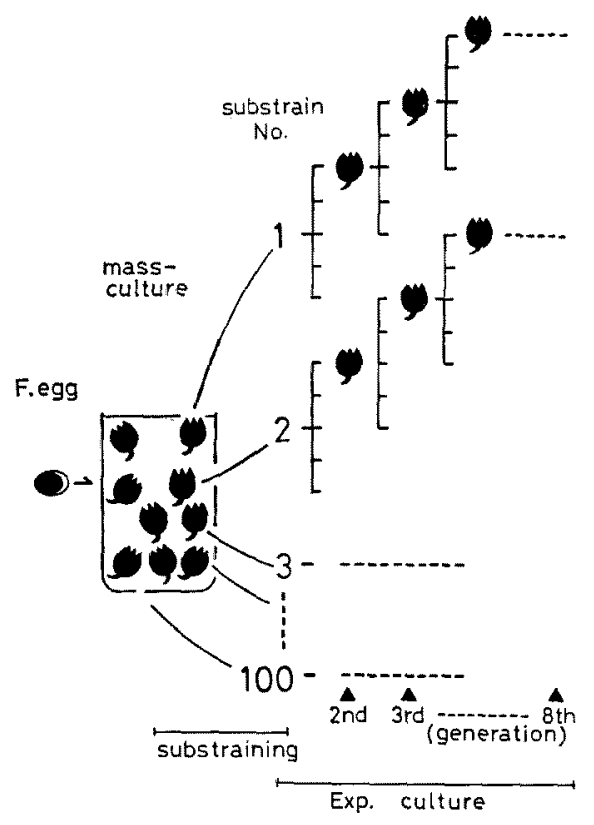

Fig. 1. Experimental method - Substraining and successive culture.

avoid the interferrence of physiological conversion of live food, such as green algae, in different temperature conditions.

A hundred females were picked up from the massculture of the aforesaid strain, and alloted individually to a hundred substrains. Each twenty or fourty substrains were incubated in four temperature conditions $(15,20,25$ and 30 , each \pm $0.5^{\circ} \mathrm{C}$ ) down to seventh or eighth generation in

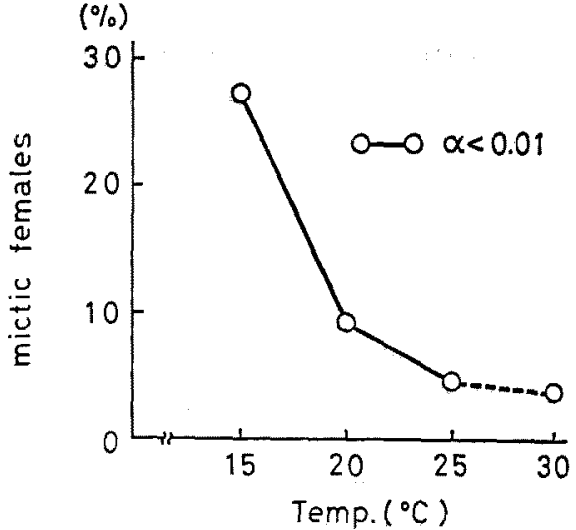

Fig. 3. Rate of mictic female production under four temperature conditions.

total darkness after substraining. These succesive cultures were inevitable particularly in the initial stage of the experiment in order to distingish the mictic female production whether it was induced by the experimental condition itself or the results of transferrence into different temperature.

Any rotifer was cultured individually in a glass tube containing $0.1 \mathrm{~m} l$ medium, and transferred into new medium at regular intervals (T). Then a newly hatched offspring was received into another culture tube. The first daughter of each generation was employed as the mother of next generation and cultured till she produced five offsprings (Fig. 1), In case that five offsprings could not be obtained from the mother because of sterility, death or being mictic, the next available daughter was employed.

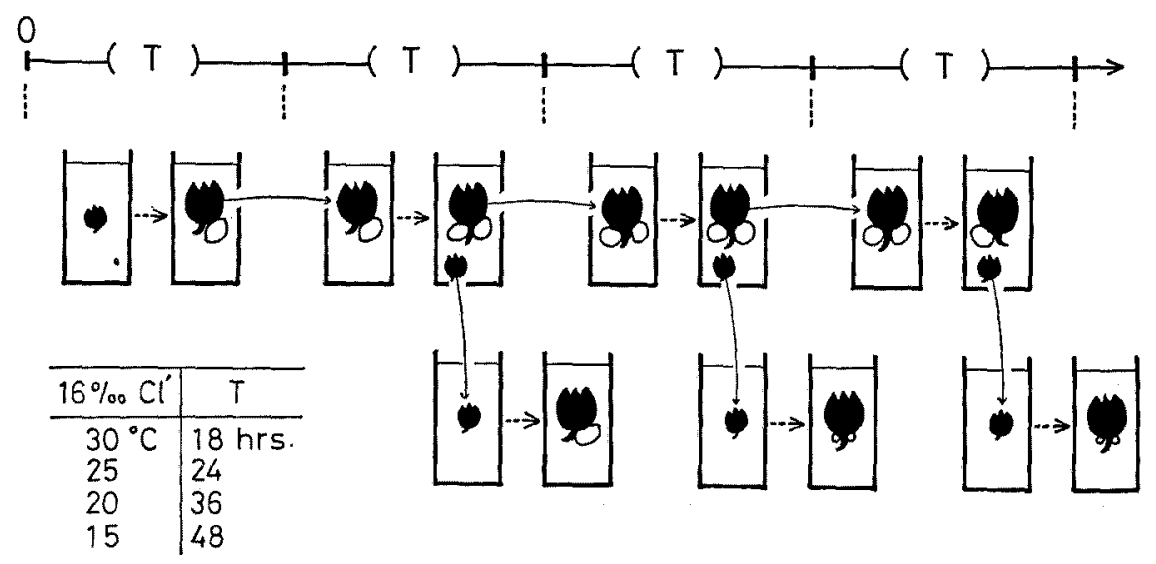

Fig. 2. Experimental method--Nullifying procedure of density effect.

Medium change and separation of neonate were made every " $T$ " which corresponds to the time required for each hatching and maturing. 
Table 1. Ratio of mictic females appearing among the first five offsprings of each generation under four temperature conditions

\begin{tabular}{|c|c|c|c|c|c|c|c|c|c|c|c|c|c|}
\hline \multirow{2}{*}{\multicolumn{2}{|c|}{$\begin{array}{c}15^{\circ} \mathrm{C} \\
\text { Substrain }\end{array}$}} & \multicolumn{4}{|c|}{$\begin{array}{c}\text { Generation after } \\
\text { the Exp. was started }\end{array}$} & \multirow[b]{2}{*}{ Total } & \multirow{2}{*}{$\begin{array}{c}25^{\circ} \mathrm{C} \\
\text { Substrain }\end{array}$} & \multicolumn{5}{|c|}{$\begin{array}{l}\text { Generation after } \\
\text { the Exp. was started }\end{array}$} & \multirow[b]{2}{*}{ Total } \\
\hline & & 4 & 5 & 6 & 7th & & & 4 & 5 & 6 & 7 & 8 th & \\
\hline 01 & & $3 / 5$ & $0 / 3$ & $1 / 3$ & $0 / 2$ & $4 / 13$ & 41 & $0 / 4$ & $0 / 4$ & $0 / 5$ & $1 / 5$ & $0 / 5$ & $1 / 23$ \\
\hline 02 & & $0 / 3$ & $2 / 5$ & $1 / 5$ & $0 / 4$ & $3 / 17$ & 42 & $0 / 5$ & $0 / 3$ & $0 / 5$ & $0 / 3$ & $0 / 2$ & $0 / 18$ \\
\hline 03 & & $0 / 3$ & $0 / 3$ & $1 / 4$ & $0 / 4$ & $3 / 14$ & 43 & $0 / 2$ & $0 / 2$ & $0 / 4$ & $0 / 5$ & $1 / 4$ & $1 / 17$ \\
\hline 04 & & $0 / 4$ & $1 / 3$ & $1 / 3$ & $1 / 3$ & $3 / 13$ & 44 & $0 / 2$ & $0 / 5$ & $0 / 4$ & $0 / 1$ & $0 / 3$ & $0 / 15$ \\
\hline 05 & & $1 / 5$ & $3 / 5$ & $0 / 3$ & $1 / 4$ & $5 / 17$ & 45 & $0 / 4$ & $1 / 3$ & $0 / 3$ & $0 / 3$ & $2 / 4$ & $3 / 17$ \\
\hline 06 & & $0 / 1$ & $2 / 5$ & $1 / 2$ & $2 / 3$ & $5 / 11$ & 46 & $0 / 3$ & $0 / 5$ & $0 / 5$ & $0 / 3$ & $0 / 5$ & $0 / 21$ \\
\hline 07 & & $0 / 2$ & $1 / 1$ & & & $1 / 3$ & 47 & $1 / 5$ & $0 / 3$ & $0 / 5$ & $1 / 5$ & $0 / 3$ & $2 / 21$ \\
\hline 08 & & $1 / 5$ & $0 / 4$ & $3 / 4$ & $0 / 1$ & $4 / 14$ & 48 & $1 / 2$ & $1 / 5$ & $0 / 4$ & $0 / 3$ & $0 / 4$ & $2 / 18$ \\
\hline 09 & & $1 / 1$ & & & & 1/ 1 & 49 & $0 / 4$ & $0 / 2$ & $0 / 3$ & $0 / 2$ & $0 / 2$ & $0 / 13$ \\
\hline 10 & & $2 / 5$ & $2 / 4$ & $0 / 5$ & $0 / 3$ & $4 / 17$ & 50 & $1 / 4$ & $0 / 3$ & $0 / 5$ & $0 / 2$ & $0 / 5$ & $1 / 19$ \\
\hline 11 & & $1 / 3$ & $1 / 3$ & $1 / 4$ & $1 / 2$ & $4 / 12$ & 51 & $0 / 2$ & $0 / 2$ & $0 / 5$ & $0 / 3$ & $0 / 2$ & $0 / 14$ \\
\hline 12 & & $2 / 5$ & $3 / 4$ & $0 / 1$ & $1 / 1$ & $6 / 11$ & 52 & $0 / 4$ & $0 / 2$ & $0 / 5$ & $0 / 4$ & $0 / 3$ & $0 / 18$ \\
\hline 13 & & $0 / 5$ & $0 / 3$ & $1 / 3$ & & $1 / 11$ & 53 & $1 / 1$ & & & & & $1 / 1$ \\
\hline 14 & & $0 / 5$ & $2 / 5$ & $2 / 5$ & $2 / 2$ & $6 / 17$ & 54 & $0 / 4$ & $0 / 5$ & $0 / 3$ & $0 / 5$ & $0 / 5$ & $0 / 22$ \\
\hline 15 & & $0 / 5$ & $2 / 3$ & & & $2 / 8$ & 55 & $0 / 5$ & $0 / 3$ & $1 / 5$ & $1 / 4$ & $1 / 4$ & $3 / 21$ \\
\hline 16 & & $0 / 4$ & $1 / 2$ & $1 / 2$ & $1 / 2$ & $3 / 10$ & 56 & $0 / 5$ & $0 / 5$ & $1 / 4$ & $0 / 5$ & $1 / 3$ & $2 / 22$ \\
\hline 17 & & $0 / 4$ & & & & $0 / 4$ & 57 & $0 / 4$ & $1 / 5$ & $0 / 3$ & $0 / 3$ & $0 / 2$ & $1 / 17$ \\
\hline 18 & & $0 / 3$ & $1 / 5$ & $0 / 2$ & & $1 / 10$ & 58 & $0 / 3$ & $0 / 4$ & $0 / 4$ & $0 / 5$ & $0 / 1$ & $0 / 17$ \\
\hline 19 & & $1 / 3$ & $1 / 5$ & $0 / 3$ & $1 / 1$ & $3 / 12$ & 59 & $0 / 5$ & $0 / 3$ & $0 / 3$ & $0 / 5$ & $0 / 5$ & $0 / 21$ \\
\hline \multirow[t]{5}{*}{20} & & $0 / 2$ & $0 / 1$ & & & $0 / 3$ & 60 & $0 / 2$ & $0 / 5$ & $0 / 5$ & $0 / 4$ & $0 / 4$ & $0 / 20$ \\
\hline & & & & & Total & $59 / 218$ & 61 & $0 / 1$ & $1 / 5$ & $0 / 4$ & $1 / 4$ & & $2 / 14$ \\
\hline & \multirow{4}{*}{\multicolumn{5}{|c|}{$\begin{array}{l}\text { Generation after } \\
\text { the Exp. was started }\end{array}$}} & \multirow{6}{*}{ Total } & 62 & $0 / 5$ & $0 / 3$ & $1 / 5$ & $2 / 5$ & & $3 / 18$ \\
\hline & & & & & & & 63 & $0 / 4$ & $0 / 5$ & $0 / 4$ & $0 / 5$ & & $0 / 18$ \\
\hline & & & & & & & 64 & $0 / 4$ & $0 / 4$ & $0 / 3$ & $0 / 5$ & & $0 / 16$ \\
\hline $20^{\circ} \mathrm{C}$ & & & & & & & 65 & $0 / 5$ & $0 / 4$ & $0 / 5$ & $0 / 4$ & & $0 / 18$ \\
\hline Substrain & 4 & 5 & 6 & 7 & 8th & & 66 & $0 / 5$ & $0 / 4$ & $0 / 4$ & $0 / 4$ & & $0 / 17$ \\
\hline & & & & & & & 67 & $0 / 4$ & $0 / 3$ & $0 / 5$ & $0 / 4$ & & $0 / 16$ \\
\hline 21 & $0 / 3$ & $0 / 5$ & $1 / 5$ & $0 / 5$ & $1 / 4$ & $2 / 22$ & 68 & $0 / 5$ & $0 / 4$ & $0 / 4$ & $0 / 5$ & & $0 / 18$ \\
\hline 22 & $0 / 3$ & $0 / 4$ & $0 / 4$ & $1 / 4$ & $0 / 3$ & $1 / 18$ & 69 & $0 / 5$ & $0 / 4$ & $1 / 5$ & $0 / 5$ & & $1 / 19$ \\
\hline 23 & $0 / 3$ & $1 / 5$ & $0 / 3$ & $1 / 5$ & $1 / 3$ & $3 / 19$ & 70 & $0 / 5$ & $0 / 5$ & $0 / 5$ & $0 / 5$ & & $0 / 20$ \\
\hline 24 & $1 / 5$ & $1 / 5$ & $2 / 5$ & $0 / 4$ & $0 / 2$ & $4 / 21$ & 71 & $0 / 5$ & $0 / 5$ & $0 / 5$ & $0 / 3$ & & $0 / 18$ \\
\hline 25 & $0 / 5$ & $1 / 3$ & $0 / 5$ & $0 / 5$ & $0 / 4$ & $1 / 22$ & 72 & $0 / 3$ & $0 / 4$ & $0 / 5$ & $0 / 5$ & & $0 / 17$ \\
\hline 26 & $0 / 4$ & $0 / 5$ & $1 / 2$ & $0 / 1$ & $0 / 1$ & $1 / 13$ & 73 & $0 / 4$ & $0 / 2$ & $0 / 5$ & $0 / 4$ & & $0 / 15$ \\
\hline 27 & $0 / 5$ & $0 / 5$ & $1 / 3$ & $0 / 5$ & $0 / 5$ & $1 / 23$ & 74 & $1 / 5$ & $0 / 5$ & $0 / 4$ & $0 / 1$ & & $1 / 15$ \\
\hline 28 & $0 / 5$ & $2 / 4$ & $0 / 5$ & $0 / 5$ & $0 / 3$ & $2 / 22$ & 75 & $0 / 3$ & $0 / 5$ & $0 / 4$ & $0 / 5$ & & $0 / 17$ \\
\hline 29 & $0 / 5$ & $1 / 4$ & $0 / 2$ & $1 / 1$ & & $2 / 12$ & 76 & $0 / 3$ & $1 / 4$ & $0 / 5$ & $0 / 4$ & & $1 / 16$ \\
\hline 30 & $0 / 4$ & $0 / 2$ & $0 / 3$ & $0 / 2$ & $0 / 2$ & $0 / 13$ & 77 & $0 / 1$ & $0 / 3$ & $0 / 4$ & $1 / 3$ & & $1 / 11$ \\
\hline 31 & $0 / 5$ & $0 / 5$ & $0 / 1$ & $0 / 1$ & & $0 / 12$ & 78 & $0 / 5$ & $0 / 5$ & $0 / 5$ & $0 / 3$ & & $0 / 18$ \\
\hline 32 & $0 / 1$ & $1 / 2$ & $0 / 2$ & $0 / 2$ & $0 / 5$ & $1 / 12$ & 79 & $0 / 3$ & $1 / 5$ & $0 / 4$ & $0 / 4$ & & $1 / 16$ \\
\hline 33 & $0 / 4$ & $0 / 4$ & $0 / 1$ & $0 / 1$ & $0 / 1$ & $0 / 11$ & 80 & $1 / 5$ & $0 / 5$ & $0 / 4$ & $2 / 3$ & & $3 / 17$ \\
\hline 34 & $1 / 4$ & $0 / 5$ & $1 / 4$ & $0 / 4$ & $0 / 5$ & $2 / 22$ & & & & & & Total & $30 / 689$ \\
\hline 35 & $0 / 4$ & $1 / 4$ & $0 / 3$ & $1 / 5$ & $1 / 3$ & $3 / 19$ & & & & & & & \\
\hline 36 & $0 / 2$ & $0 / 5$ & $2 / 5$ & $0 / 5$ & $0 / 5$ & $2 / 22$ & & & & & & & \\
\hline 37 & $0 / 1$ & $1 / 4$ & $0 / 5$ & $0 / 3$ & $0 / 1$ & $1 / 14$ & & & & & & & \\
\hline 38 & $0 / 3$ & $1 / 3$ & $0 / 5$ & $0 / 4$ & $1 / 4$ & $2 / 19$ & & & & & & & \\
\hline 39 & $0 / 1$ & $0 / 2$ & $2 / 2$ & & & $2 / 5$ & & & & & & & \\
\hline \multirow[t]{2}{*}{40} & $0 / 1$ & $0 / 1$ & $0 / 1$ & $0 / 1$ & $0 / 1$ & $0 / 5$ & & & & & & & \\
\hline & & & & & Total & $30 / 326$ & & & & & & & \\
\hline
\end{tabular}


Table 1. (continues)

\begin{tabular}{|c|c|c|c|c|c|c|}
\hline \multirow{2}{*}{$\begin{array}{c}30^{\circ} \mathrm{C} \\
\text { Substrain }\end{array}$} & \multicolumn{5}{|c|}{$\begin{array}{l}\text { Generation after } \\
\text { the Exp. was started }\end{array}$} & \multirow[b]{2}{*}{ Total } \\
\hline & 4 & 5 & 6 & 7 & 8th & \\
\hline 81 & $0 / 5$ & $1 / 5$ & $2 / 5$ & $0 / 3$ & $0 / 4$ & $3 / 22$ \\
\hline 82 & $0 / 4$ & $1 / 4$ & $0 / 5$ & $0 / 2$ & $0 / 3$ & $1 / 18$ \\
\hline 83 & $0 / 5$ & $0 / 5$ & $0 / 4$ & $0 / 3$ & $0 / 4$ & $0 / 21$ \\
\hline 84 & $1 / 5$ & $1 / 4$ & $1 / 4$ & $0 / 3$ & $0 / 5$ & $3 / 21$ \\
\hline 85 & $0 / 5$ & $0 / 3$ & $0 / 5$ & $0 / 5$ & $2 / 2$ & $2 / 20$ \\
\hline 86 & $0 / 5$ & $0 / 5$ & $0 / 5$ & $0 / 2$ & $0 / 4$ & $0 / 21$ \\
\hline 87 & $0 / 5$ & $0 / 5$ & $0 / 5$ & $0 / 2$ & $0 / 4$ & $0 / 21$ \\
\hline 88 & $0 / 4$ & $0 / 4$ & $0 / 5$ & $0 / 4$ & $0 / 5$ & $0 / 22$ \\
\hline 89 & $0 / 3$ & $0 / 3$ & $1 / 5$ & $0 / 3$ & $0 / 4$ & $1 / 18$ \\
\hline 90 & $0 / 5$ & $0 / 4$ & $0 / 4$ & $1 / 5$ & $0 / 5$ & $1 / 23$ \\
\hline 91 & $0 / 5$ & $0 / 3$ & $0 / 4$ & $0 / 5$ & $0 / 3$ & $0 / 20$ \\
\hline 92 & $0 / 4$ & $0 / 4$ & $0 / 4$ & $0 / 4$ & $1 / 5$ & $1 / 21$ \\
\hline 93 & $0 / 5$ & $0 / 4$ & $1 / 5$ & $1 / 3$ & $0 / 2$ & $2 / 19$ \\
\hline 94 & $0 / 5$ & $0 / 3$ & $0 / 5$ & $0 / 3$ & $0 / 3$ & $0 / 19$ \\
\hline 95 & $0 / 5$ & $0 / 4$ & $0 / 5$ & $0 / 5$ & $0 / 3$ & $0 / 22$ \\
\hline 96 & $0 / 1$ & $0 / 5$ & $0 / 5$ & $0 / 3$ & $0 / 4$ & $0 / 18$ \\
\hline 97 & $0 / 5$ & $0 / 5$ & $0 / 3$ & $0 / 5$ & $0 / 3$ & $0 / 21$ \\
\hline 98 & $1 / 5$ & $0 / 5$ & $0 / 5$ & $0 / 4$ & $0 / 3$ & $0 / 22$ \\
\hline 99 & $0 / 4$ & $0 / 5$ & $0 / 4$ & $0 / 3$ & $0 / 2$ & $0 / 18$ \\
\hline \multirow[t]{2}{*}{100} & $0 / 5$ & $0 / 5$ & $1 / 4$ & $0 / 4$ & $0 / 5$ & $1 / 23$ \\
\hline & & & & & Total & $15 / 410$ \\
\hline
\end{tabular}

The denominator means total No. of offsprings which could be discriminated mictic or amictic.

Female type was discriminated by egg size. An amictic female lays a diploid egg which is larger than a haploid egg layed by a mictic female.

\section{Nullifying the Density Effect}

Mictic female production in $B$. plicatilis is a density dependent phenomenon as in the case of $B$. calyciforus, ${ }^{\text {,) }}$ and the substance of the density effect was thought to be metabolites accumulating in culture medium. It may arise that the experimental condition of temperature may degenerate into density effect because of the difference in metabolic level, It is resolved, however, by means of renewing culture medium at regular intervals which are proportional to metabolic rate (Fig. 2). In this study, the interval was decided to the time required to get hatching of a parthenogenetic egg, which was $48,36,24$ and $18 \mathrm{~h}$. at 15 , 20,25 and $30^{\circ} \mathrm{C}$ respectively. These values were calculated with $\mathrm{Q}_{10}$ law? from the observation at $25^{\circ} \mathrm{C}$.

\section{Results and Discussion}

Table 1 shows the ratio of mictic females to the total number of individuals which could be dis-
Table 2. Numbers of mictic and amictic females appearing under four temperature conditions

\begin{tabular}{|c|c|c|c|c|c|}
\hline \multirow{2}{*}{ Female type } & \multicolumn{4}{|c|}{ Temp. experimented } & \multirow{2}{*}{ Total } \\
\hline & 15 & 20 & 25 & $30^{\circ} \mathrm{C}$ & \\
\hline Mictic & 59 & 30 & 30 & 15 & 134 \\
\hline Amictic & 159 & 296 & 659 & 395 & 1509 \\
\hline Total & 218 & 326 & 689 & 410 & 1643 \\
\hline \multirow[t]{2}{*}{ Mictic $(\%)$} & 27.1 & 9.2 & 4.4 & 3.7 & \\
\hline & & $.01 \alpha$ & 60.01 & N.S. & \\
\hline
\end{tabular}

tinguished whether mictic or amictic among the first five offsprings of each generation. The deta from the 2 nd and 3 rd generations were rejected in view of the previous report ${ }^{4)}$ where rotifers made bisexual reproduction rate down over a few generation after they had been transferred into a new culture condition. On the 4th generations of 15 and $20^{\circ} \mathrm{C}$ groups, the appearance rates of mictic females are lower than those of later generations, which shows the lowering phase has not recovered yet. As to 25 and $30^{\circ} \mathrm{C}$ groups, the phenomenon is not obvious because of the low inductivity of mictic females.

The numbers of mictic and amictic females were summed up in Table 2. As the result of $x^{2}$-test between adjoining two conditions, significant differences are in evidence below $25^{\circ} \mathrm{C}$, but it is not in existence between 25 and $30^{\circ} \mathrm{C}$ groups. Considering that $B$. plicatilis reproduces normally within the temperature from 15 to $30^{\circ} \mathrm{C}$, it is given as a conclusion that bisexual reproduction rate increases in lower temperature below $25^{\circ} \mathrm{C}$.

Generally in animals and plants, temperature has substancially two meanings. One is the induction of response specific to a certain temperature, and another is that caused by its fluctuation. In genus Brachionus, Halbach and HalBaCH-KeUP ${ }^{9)}$ reported that a fluctuation of temperature induced bisexual reproduction in $B$. calyciforus, B. rubens and $B$. angularis, contrary to the experiment on $B$. rubens by LADERMAN and GUTTMAN. ${ }^{9)}$ As to $B$. plicatilis, as stated before, it exhibits a temporary lowering of mictic female production for a few generations just after the rearing condition is changed. Consequently, positive induction of bisexual reporduction caused by fluctuation of temperature could not be recognized through our methods. 


\section{References}

1) A. Hino and R. Hirano: Kagaku to Seibutsu, 13, 516-521 (1975).

2) M. Pozuelo: Publ. Te'c. Junta Est. Pesca, 11, $401-416$ (1976).

3) A. Hino and R. Hirano: Bull. Japan. Soc. Sci. Fish., 42, 1093-1099 (1976).

4) A. Hino and R. Hirano: Bull. Japan. Soc. Sci. Fish., 43, 1147-1155 (1977).
5) S. Imamura, S. AdAchI and H. Tojo: Saibai Gyogyo Gijutsu Kaihatsu Kenkyu, 8, 53-61 (1979).

6) J. Gilbert: J. Exp. Zool., 153, 113-123 (1964).

7) K. Hirayama and T. Kusano: Bull. Japan. Soc. Sci. Fish., 38, 1357-1363 (1972).

8) Halbach, Udo, and G. Halbach-Keup: Oecologia, 9, 203-214 (1972).

9) A. Laderman and H. Guttman: J. Exp. Zool, 152, 5-11 (1963). 